\title{
Preeclampsia and Stroke: Risks during and after Pregnancy
}

\author{
Cheryl Bushnell ${ }^{1,2}$ and Monique Chireau ${ }^{3}$ \\ ${ }^{1}$ Department of Neurology, Medical Center Boulevard, Wake Forest University Health Sciences, Winston Salem, NC 27157, USA \\ ${ }^{2}$ Stroke Center Wake Forest University Baptist Medical Center and Women's Health Center of Excellence for Research, Leadership, \\ and Education, Wake Forest University Health Sciences, Medical Center Boulevard, Winston Salem, NC 27157, USA \\ ${ }^{3}$ Department of Obstetrics and Gynecology, Duke University Medical Center, Durham, NC 27710, USA
}

Correspondence should be addressed to Cheryl Bushnell, cbushnel@wfubmc.edu

Received 14 October 2010; Accepted 13 December 2010

Academic Editor: Halvor Naess

Copyright ( 2011 C. Bushnell and M. Chireau. This is an open access article distributed under the Creative Commons Attribution License, which permits unrestricted use, distribution, and reproduction in any medium, provided the original work is properly cited.

\begin{abstract}
Preeclampsia and stroke are significantly related, both pathologically and temporally (across the life span) in women. Cerebrovascular events can complicate preeclampsia, and can also manifest later in life. A history of preeclampsia is associated with long-term risk for hypertension, stroke, and heart disease. Cerebrovascular complications occur in only a small proportion of women with severe preeclampsia, but with high morbidity and mortality. Endothelial dysfunction and impaired cerebral autoregulation, and severe hypertension in the setting of preeclampsia are likely the cause of many strokes during pregnancy. The relationship between preeclampsia and stroke involves shared risk factors for both disorders, including chronic endothelial dysfunction and increased risk for long-term hypertension following preeclampsia (one of the major risk factors for stroke). This overlap provides insights into underlying pathophysiology and potential preventive strategies for both preeclampsia and stroke. For example, aspirin may prevent both disorders. The current review will describe the current data regarding these relationships and suggest future research to investigate remaining knowledge gaps. These are important topics for neurologists, who are likely to be involved with the care of severely ill preeclamptic patients with neurologic complications, as well as women at increased risk of stroke due to a history of preeclampsia.
\end{abstract}

\section{Introduction}

While stroke is a leading cause of death in postmenopausal women, it is a relatively uncommon occurrence during pregnancy. However, its morbidity and mortality are disproportionately high, especially in young women and newborn or unborn children. The incidence of stroke during pregnancy including cerebral venous thrombosis ranges from 10 to $34 / 100,000$ deliveries [1-3]. One of the most common risk factors for stroke in pregnancy, particularly postpartum, is preeclampsia/eclampsia $[2,3]$. In the most recent Nationwide Inpatient Sample epidemiological study of stroke in pregnancy, preeclampsia/eclampsia was associated with a 4fold increased risk of stroke [2]. Preeclampsia is potentially of interest to neurologists because it shares common pathophysiology and risk factors with stroke, including endothelial dysfunction, dyslipidemia, hypertension, hypercoagulability, and abnormal cerebral vasomotor reactivity. It is not surprising then, that a history of preeclampsia during pregnancy would lead to an increased risk of stroke later in life, as well [4]. The purpose of this review is to describe what is currently known about the epidemiology of preeclampsia as it related to risk factors for stroke during pregnancy and in later life. We will also identify areas where further research is needed.

\section{The Spectrum of Preeclampsia/Eclampsia}

Preeclampsia is classified as one of the hypertensive disorders of pregnancy. It is generally defined as new onset hypertension with proteinuria during pregnancy, although the term is also used to describe worsening blood pressure and proteinuria in women with a history of chronic prepregnancy 
hypertension [5]. The maternal syndrome consists of hypertension and proteinuria, which may also include multisystem abnormalities such as hemolysis, elevated liver enzymes, or low platelets (HELLP) syndrome; disseminated intravascular coagulation (DIC); acute renal failure; seizures; pulmonary edema [6]. The fetal syndrome includes fetal growth restriction, small-size-for-gestational-age (SGA), reduced amniotic fluid, and placental insufficiency leading to fetal hypoxia and hypoperfusion. Preeclampsia can occur near or before term, with or without fetal complications [6].

Preeclampsia is categorized as mild or severe. Mild preeclampsia is defined as blood pressure of at least $140 \mathrm{~mm} \mathrm{Hg}$ systolic or at least $90 \mathrm{~mm} \mathrm{Hg}$ diastolic on at least two occasions and at least 4 to 6 hours apart after the 20th week of gestation in women without prepregnancy hypertension, with proteinuria (defined as $\geq 300 \mathrm{mg}$ protein in a 24 hour urine specimen) [6]. Severe preeclampsia is defined as blood pressure at least 160 or $170 \mathrm{~mm} \mathrm{Hg}$ systolic, $110 \mathrm{~mm} \mathrm{Hg}$ diastolic, or both, accompanied by $\geq 5 \mathrm{~g}$ of proteinuria per day. When symptoms such as severe headache, visual disturbance, epigastric pain, vomiting, multiorgan system involvement, fetal morbidity or mortality, onset before 34 or 35 weeks or eclampsia (seizures) are present, preeclampsia is also classified as severe (see Table 1). Eclampsia, defined as tonicclonic seizures in a pregnant or recently delivered woman not attributable to other causes than preeclampsia or gestational hypertension, complicates about 1 to $2 \%$ of all cases of severe preeclampsia [7]. It is important to note that published guidelines for preeclampsia diagnostic criteria vary among different professional societies [7].

\section{Epidemiology of Preeclampsia}

Preeclampsia is part of the spectrum of hypertensive disorders in pregnancy, which also includes gestational hypertension $(\mathrm{GH})$. GH is defined as elevated blood pressure of at least $140 \mathrm{~mm} \mathrm{Hg}$ systolic or at least $90 \mathrm{~mm} \mathrm{Hg}$ diastolic without proteinuria in a woman after 20 weeks' gestation, which resolves postpartum [10]. Hypertensive disorders in pregnancy affect $8 \%-12 \%$ of all pregnancies, and this rate is even higher in developing countries. About three quarters of cases of preeclampsia in women are classified as mild, with onset at or near-term [6]. Adverse outcomes are generally rare in mild preeclampsia. However, it is important to note that mild preeclampsia is a retrospective diagnosis. Mild hypertension in pregnancy can evolve quickly and unpredictably to fulminant severe preeclampsia. African American women appear to be at higher risk for the development of preeclampsia, although it is not clear whether this relationship is due to a higher prevalence of hypertension in African American women or other risk factors. Women living at high altitude are also known to be at higher risk for preeclampsia.

\section{Risk Factors for Preeclampsia}

Multiple risk factors for preeclampsia have been identified through clinical and large cohort studies. Many of these risk factors overlap with those for stroke and cardiovascular disease (CVD), such as family history, prepregnancy hypertension, diabetes, obesity and insulin resistance, preexisting thrombophilia, renal disease, and collagen-vascular disease [11]. Some pregnancy-specific and maternal risk factors include maternal infections, extremes of maternal age, multiple gestation, maternal low birthweight, hydrops fetalis, and preeclampsia in a prior pregnancy $[7,12]$ (see Duckitt et al. for comprehensive review). An unusual protective factor against preeclampsia is maternal smoking, for unclear reasons.

Immune and paternal factors may also affect women's risk for preeclampsia. In a large birth cohort in Norway, the rate of preeclampsia was $3.4 \%$ in women with their first pregnancy, whereas the rate of preeclampsia in the second pregnancy with the same father was $1.7 \%$; other studies have noted an increase in the risk for preeclampsia with a change in paternity, limited sperm exposure with the same partner, or increased duration of time between pregnancies [13]. These findings suggested that an immune tolerance mechanism contributes to the pathogenesis of preeclampsia [14]. This theory has recently been challenged by findings that preterm preeclampsia is more likely to recur than term preeclampsia, which is not consistent with an immune-mediated mechanism. Preterm preeclampsia is also more likely to be associated with long-term CVD, which implicates maternal factors as being important in its pathogenesis [15]. Other paternal factors have been shown to contribute to the risk for preeclampsia. In the Norwegian study cited above, men who fathered one preeclamptic pregnancy were 1.8 -fold as likely to father a preeclamptic pregnancy in a different woman (OR 1.8; 95\% CI 1.22.6) [14]. Preeclampsia has a clear genetic component. In an intergenerational study of paternal and maternal contributions to the risk of developing preeclampsia, women whose mothers had preeclampsia were more likely to have the condition in their own pregnancies. Men born after a pregnancy complicated by preeclampsia were more likely to father a pregnancy complicated by preeclampsia, and for both women and men, familial preeclampsia was associated with more severe preeclampsia in the index pregnancy [16].

A novel risk factor for preeclampsia may be migraine. There is a growing body of literature to suggest an association between migraine and preeclampsia, although no cause and effect has been established. The symptomatology of both conditions may overlap, since severe preeclampsia may involve headache with visual scotoma [7], similar to migraine with aura. A review of all cross-sectional, casecontrol, and cohort studies published prior to 2006 revealed 10 studies that examined the association between migraine and preeclampsia, 8 of which showed a positive and significant relationship [17]. Since then, at least 2 additional studies have been published. In a large study of delivery hospitalization discharges in the Nationwide Inpatient Sample, over 34,000 women had a primary or secondary discharge diagnosis of peripartum migraine [18]. The association between preeclampsia and migraine (OR 2.29, 95\% CI 2.13-2.46) was so robust that preeclampsia diagnosis codes had to be excluded in order to analyze the association 
TABLE 1: Criteria for classification of severe preeclampsia $[8,9]$.

\begin{tabular}{|c|c|}
\hline Severity Criteria & Recommendations from SOGC and ASH \\
\hline Gestational age at onset & Less than 34 to 35 weeks' gestation \\
\hline Maternal symptoms & $\begin{array}{l}\text { Persistent or new/unusual headache; visual disturbances; persistent abdominal or right upper quadrant } \\
\text { pain; severe nausea or vomiting; chest pain or dyspnea }\end{array}$ \\
\hline $\begin{array}{l}\text { Maternal signs of end-organ } \\
\text { dysfunction }\end{array}$ & $\begin{array}{l}\text { Eclampsia; severe hypertension (>160/110 mm Hg); pulmonary edema; suspected placental abruption; } \\
\text { severe diastolic hypertension (>110 } \mathrm{mm} \mathrm{Hg}) \text {; heavy proteinuria }(3 \mathrm{~g} / \text { day) or oliguria }\end{array}$ \\
\hline $\begin{array}{l}\text { Abnormal maternal laboratory } \\
\text { testing }\end{array}$ & $\begin{array}{l}\text { Raised serum creatinine; increased AST, ALT, or LDH with symptoms; platelet count }<100 \times 10^{9} / \mathrm{L} \text {; or } \\
\text { serum albumin }<20 \mathrm{~g} / \mathrm{L} \text {; decreased glomerular filtration rate }\end{array}$ \\
\hline Fetal morbidity or mortality & $\begin{array}{l}\text { Oligohydramnios; intrauterine growth restriction; absent or reversed end-diastolic flow in umbilical } \\
\text { artery by Doppler velocimetry; intrauterine fetal death; any fetal morbidity (non-reassuring fetal testing) }\end{array}$ \\
\hline
\end{tabular}

SOGC: Society of Obstetricians and Gynaecologists of Canada; ASH: American Society of Hypertension; AST: aspartate transaminase; ALT: alanine transaminase; LDH: lactate dehydrogenase.

between migraine and stroke [18]. A case control study from Peru showed an incrementally stronger relationship between preeclampsia and any headache before or during pregnancy (OR 2.4, 95\% CI 1.7-3.3), migraine prior to pregnancy (OR 3.5, 95\% CI 1.9-6.4), and migraine during pregnancy (OR 4.0, 95\% CI 1.9-9.2) [19]. The mechanism for this association is currently unknown, but may relate to endothelial dysfunction, widely recognized as important in the pathophysiology of both conditions [17-20].

\section{Risks of Cerebrovascular Disease in Women with Preeclampsia}

Fewer than $1 \%$ of women with preeclampsia suffer from stroke during the puerperium (the first six weeks postpartum). However, among women with stroke during pregnancy, preeclampsia/eclampsia is an important risk factor for both ischemic and hemorrhagic strokes. For example, in a French case series of 31 women with stroke during pregnancy, about half $(n=15)$ were ischemic, with preeclampsia/eclampsia accounting for 6 cases or $47 \%$. Of those with hemorrhagic strokes, 7 (44\%) had eclampsia, in addition to HELLP syndrome or disseminated intravascular coagulation [21]. A Canadian case series reported that 7 of 34 or $21 \%$ of women with peripartum stroke had preeclampsia [1]. In Taiwan, 7 of 19 or $37 \%$ of women with peripartum stroke had preeclampsia/eclampsia or hypertensive intracerebral hemorrhage [22]. These single-center case series are limited due to the small sample sizes, but larger cohort studies have confirmed this association. The Nationwide Inpatient Sample analysis from 2000 to 2001 reported that preeclampsia was associated with a 4 -fold increase in stroke during pregnancy (OR 4.4; 95\% CI 3.6-5.4) [2]. A limitation of this study, as with most administrative databases, was the need to rely on correct assignment of ICD-9 codes and the inability to review medical records to collect individual level patient details.

Hemorrhagic stroke is the most common stroke type associated with pregnant or postpartum women with preeclampsia/eclampsia. Data from the Nationwide Inpatient Sample examining women aged 15 to 44 showed that most cases of pregnancy-associated hemorrhagic strokes occurred postpartum [23]. The risk factors independently associated with hemorrhagic stroke included preexisting hypertension
(OR 2.6; 95\% CI 1.34-5.07), gestational hypertension (OR 2.41; 95\% CI 1.62-3.59), and preeclampsia/eclampsia (OR 10.4; 95\% CI 8.3-13.0) [23]. The in-hospital mortality was $20 \%$ [23]. In a detailed series of 27 women with preeclampsia and subsequent stroke, $25(89 \%)$ had hemorrhagic and $2(7 \%)$ had ischemic stroke; $96 \%$ of these women had headache, $63 \%$ with nausea and vomiting, $71 \%$ had symptoms attributable to the central nervous system (e.g., focal weakness, seizure, syncope, decreased level of alertness), and $37.5 \%$ had visual problems [24]. Similar to the Nationwide Inpatient Sample analysis, the majority (57\%) of strokes occurred postpartum. Another striking feature in this series was that $96 \%$ of these women had prestroke systolic blood pressures $\geq 160 \mathrm{~mm} \mathrm{Hg}$, whereas only $21 \%$ of women had diastolic blood pressures $>105 \mathrm{~mm} \mathrm{Hg}$. Not surprisingly, given the high rate of hemorrhagic strokes, the mortality was 54\% [24]. The important message from this case series was that, in contrast to current management protocols which base treatment decisions on elevated diastolic blood pressures, women with severe preeclampsia and high isolated systolic blood pressures should be considered at high risk for hemorrhagic stroke and that antihypertensive therapy should be considered in these patients [24].

Besides ischemic and hemorrhagic stroke, preeclampsia is often associated with the syndrome of reversible posterior leukoencephalopathy syndrome (RPLS; also known as posterior reversible encephalopathy syndrome or PRES) [25]. This disorder differs pathophysiologically from ischemic or hemorrhagic stroke because it is associated with reversible vasogenic edema seen on CT or MRI, usually in the occipital or parietal lobes $[26,27]$. This disorder is thought to be similar to hypertensive encephalopathy and is associated with seizures, headaches, encephalopathy, and reversible imaging features. RPLS may occur in the setting of preeclampsia due to impaired cerebral autoregulation from endothelial damage. Large changes in blood pressure (rather than the absolute blood pressure) can then result in an imbalance of the capillary and cellular perfusion pressures, leading to vasogenic edema $[27,28]$. The treatment of this disorder includes blood pressure lowering and antiepileptics, although most patients do not require treatment for seizures beyond the acute syndrome.

Imaging in women with RPLS associated with the puerperium has been described as diffuse or multifocal segmental 
vasoconstriction (or vasospasm) [28]. As this disorder is increasingly recognized there are features that are considered atypical compared with the original definition. For example, in the largest case series of RPLS to date, over half of the patients had significant frontal involvement, in addition to $5 \%$ that included hemorrhage and confluent vasogenic edema [26]. Also, about one quarter of these cases had permanent injury, although the lesions were small on repeat MRI. The imaging abnormalities resolved completely in about two-thirds of cases in this series, which means that there are still a considerable number of patients with residual abnormalities [26].

A similar disorder that occurs in association with preeclampsia is reversible cerebral vasoconstriction syndromes (RCVS). The classical presentation of RCVS, also known as Call Fleming Syndrome, is a thunderclap headache, with or without additional neurologic signs [29]. Diagnostic imaging reveals multifocal segmental vasoconstriction of the cerebral arteries, which usually resolves within days to weeks. Besides occurring during the pregnancy or puerperium, this syndrome can occur as an idiosyncratic response to medications or illicit drugs, or without any identifiable cause. Although the pathophysiology of all of the vasoconstriction syndromes is not fully understood, it involves a disturbance in the control of cerebral vascular tone. In the setting of preeclampsia/eclampsia, the syndrome of RCVS usually occurs postpartum (often labeled postpartum angiopathy) [29], and in severe cases of eclampsia, superimposed cytotoxic edema and chronic infarction may also occur [30]. Based on imaging, this diagnosis may be difficult to differentiate from central nervous system vasculitis, thus analysis of spinal fluid may be helpful. Clinically, however, vasculitis usually involves an insidious headache rather than the thunderclap headache characteristic of RCV. The treatment of RCVS is not standardized because it is not known whether the rapid resolution of symptoms may be due to the natural history of the syndrome or therapeutic strategies that are empirically initiated. In confirmed cases with imaging, calcium channel blockers may be initiated, such as nimodipine or verapamil, although caution should be employed to maintain cerebral perfusion in watershed regions of a constricted artery [29]. Other medications have included high-dose glucocorticoids [31] or magnesium sulfate [32].

The amount of overlap between RPLS and RCVS is unclear because some of the larger case series of RPLS did not include arterial imaging $[26,27]$. We propose that there is likely to be overlap in the pathophysiology and therefore imaging characteristics of many of the cerebrovascular syndromes associated with preeclampsia/eclampsia, as shown in Figure 1. The primary difference between RPLS and RCVS is in the clinical symptoms. RCVS manifests in a thunderclap headache, whereas RPLS is more likely to include seizures and encephalopathy with nonthunderclap headache. As noted above, because many women do not undergo brain imaging, the true prevalence of cerebrovascular complications such as RPLS and RCVS is unknown. In addition, guidelines recommend recognition and treatment of severe preeclampsia, whether it includes severe hypertension or the onset of severe headache or neurologic signs of any

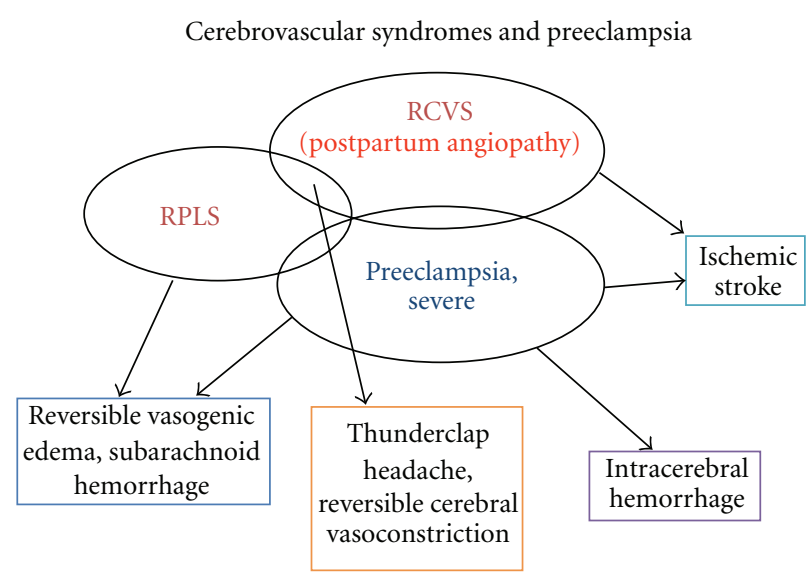

Figure 1

kind [7]. Therapeutic maneuvers such as administration of magnesium sulfate, glucocorticoids, antihypertensive therapy, or delivery of the baby may also effectively treat the reversible syndrome and its clinical symptoms, resulting in the inability to distinguish the two disorders. Because of the urgency of delivering the infant and stabilizing the mother, imaging may also not be performed commonly unless clinically indicated. Therefore, it is possible that the incidence of milder, subclinical forms of either RPLS or RCVS on imaging is much higher than currently recognized. Carefully designed pregnancy registries with detailed clinical and neurological details may provide some insights into the incidence of these disorders. In addition, noninvasive monitoring with ultrasound, such as transcranial Doppler, may also allow evaluation of the cerebral hemodynamics of severe preeclampsia, although it is currently unclear which parameters are most important to measure $[33,34]$.

\section{Proposed Pathophysiology of Preeclampsia}

The underlying cause of preeclampsia is still unknown, but there are well-recognized combinations of placental, immune, and vascular factors that lead to the condition. The placenta is central to the pathophysiology of preeclampsia, since the latter does not occur in its absence. Preeclampsia is thought to occur in 2 stages [15]. In the first stage, abnormal placentation and maternal vascular remodeling lead to placental underperfusion, hypoxia, and/or oxidative stress, with placental release of factors which causes the second stage of endothelial dysfunction and other manifestations of preeclampsia. In the first stage, defective trophoblast differentiation and immune mechanisms related to interactions between maternal $\mathrm{T}$ cells and placental cytotrophoblast are hypothesized to be involved with the inadequate placentation early in pregnancy. This leads to inadequate remodeling of the spiral arteries during the first 12 weeks of pregnancy. In the second stage, the onset of systemic endothelial activation involving imbalance between angiogenic and antiangiogenic cytokines occurs as a result of insufficient placental perfusion via the spiral arteries [35]. Another hypothesis involves abnormal syncytiotrophoblast shedding into the maternal circulation, which incites an inflammatory reaction [36]. 
Consistent with the role of angiogenesis in the pathophysiology of preeclampsia, several blood biomarkers of angiogenesis have been studied to determine their predictive value for the development of preeclampsia later in pregnancy. Soluble flt1, also known as soluble vascular endothelial growth factor receptor 1 (sVEGFR1), is a protein produced by the placenta which binds to placental growth factor (PlGF) and VEGF, both of which are proangiogenic. Levels of sFlt 1 are low in early pregnancy but rise steadily thereafter. It is hypothesized that the balance between these factors regulates placental angiogenesis, with increasing levels of sFlt1 and decreasing levels of PlGF and VEGF exerting a restraining effect on placental vascular growth via a net antiangiogenic effect [37]. Endoglin is a coreceptor for transforming growth factor beta 1 and beta 3 , which is highly expressed on endothelial and syncytiotrophoblast surfaces. Soluble endoglin is also antiangiogenic and increased in preeclampsia [38]. The combination of these biomarkers was studied using a nested case control cohort of the Calcium for Preeclampsia Prevention Trial [39]. When using a cutoff for high versus low levels of both markers, the combination of high endoglin and high sflt1:PlGF ratios at 21 to 32 weeks gestation was associated with 31.6-fold odds of preterm preeclampsia and 30.8-fold odds of term preeclampsia [39]. These markers appear to be promising for identifying women early enough during pregnancy to provide early intervention.

\section{Risks of Stroke Later in Life}

Although women with cerebrovascular manifestations of preeclampsia are thought to be out of danger when the baby is delivered, accumulated data show that women with preeclampsia are at risk for stroke and cardiovascular disease well after the postpartum period and child bearing years [40-46]. A meta-analysis investigated the link between preeclampsia and cardiovascular disease, stroke, and relevant risk factors [4]. The cumulative odds ratios nicely illustrate the association. For example, there is nearly a 4 -fold odds of developing hypertension (OR 3.7, 95\% CI 2.70-5.05) and a 2-fold risk of ischemic heart disease (OR 2.16, 95\% CI 1.862.52) in women with preeclampsia. For fatal and nonfatal stroke, the meta-analysis included 4 studies $[43,44,47,48]$ with over 64,000 women with preeclampsia and about 900 ischemic strokes. The relative risks ranged from 1.39 to 5.08. The cumulative relative risk was 2 -fold with preeclampsia (RR 2.16, 95\% CI 1.86-2.52) [4].

In addition to maternal risk, children born to mothers with preeclampsia pregnancies may also be at increased risk for neurological problems and stroke. The Helsinki Birth Cohort traced offspring of the original cohort born between 1934 and 1944 in Helsinki, Finland [49]. The hazard ratio for all forms of stroke in offspring of mothers with preeclampsia was 1.9 (95\% CI 1.2 to 3.0), and the hazard ratio was 1.4 for those born to women with pregnancies complicated by gestational hypertension (95\% CI 1.0-1.8). There was no increased risk of coronary heart disease, however. Severe preeclampsia was also associated with a reduced head circumference at birth [49].
In addition to the risk of cardiovascular events, the prevalence of cardiovascular risk factors and abnormal vascular markers appears to be higher in women with a history of preeclampsia. For example, women with a history of preeclampsia about 20 years before being studied (mean age at assessment 43 years) had higher diastolic blood pressure and were more likely to be menopausal than women without preeclampsia [50]. Although there were no differences in lipids, biomarkers of endothelial dysfunction such as intercellular adhesion molecule-1 (ICAM-1) and vascular cell adhesion molecule-1 (VCAM-1) were elevated in the preeclampsia group [50]. Other studies have shown that women with a history of preeclampsia are more likely to have higher insulin levels compared to controls [51]. However, not all studies have shown consistent differences in markers for cardiovascular risk. Vickers et al. reported no differences in levels of $\mathrm{C}$ reactive protein (CRP), von Willebrand factor, or fibrinogen in women with a history of preeclampsia or gestational hypertension compared with controls [52]. As described in the meta-analysis and other longitudinal studies, hypertension is the risk factor for cerebrovascular disease that women with a history of preeclampsia and gestational hypertension are most likely to develop $[4,43]$.

Women with a history of preeclampsia have also been shown to have abnormal endothelial function well after the puerperium. In a study of women in a minimum of 3 months following pregnancy, those with a prior history of preeclampsia had lower brachial artery flow mediated dilation responses than women with normal pregnancies $(0.9 \%$ for those with multiple preeclamptic episodes, $2.7 \%$ for single episode, and $4.7 \%$ for controls) [53]. Preeclampsia was an independent predictor of flow mediated dilation, even after adjustment for family history, cardiovascular risk factors such as cigarette smoking, lipids, glucose, and systolic blood pressure [53]. Interestingly, when brachial artery diameter in response to nitroglycerin, an endotheliumindependent response, was measured, there was no difference between groups [53]. Based on these results, preeclampsia appears to have a chronic deleterious effect on the endothelium, regardless of other risk factors. Similarly, the association between preeclampsia and impaired flow mediated dilation was also demonstrated in a separate, smaller cohort measuring flow mediated dilation 6 to 12 months postpartum [54]. Another study of women with early preeclampsia assessed 3 to 11 months postpartum reported impaired microvascular skin reactivity, whereas endothelialindependent activity was not significantly different from controls [55]. More importantly for future risk of stroke, in a study of visually evoked cerebral blood flow responses in the posterior cerebral artery measured with transcranial Doppler ultrasound, women with a history of preeclampsia have a dampened response to this stimulus [56]. Taking into account the time from preeclampsia to the testing, there also appeared to be diminishing cerebral hemodynamic function over time associated with a history of preeclampsia. Alternatively, this could represent worse function at baseline [56]. In either case these findings provide another important pathophysiologic basis for an increased risk for stroke in women with a history of preeclampsia. 


\section{The Duke Birth Database}

There are very few studies of women followed prospectively after preeclampsia other than with administrative databases, and many of the studies were limited to Caucasian race/ethnicity. We performed an Institutional Review Boardapproved search of the Duke Perinatal Health Services Outcomes Database, a medical record database of 42,263 women admitted to Duke University Medical Center from 1979 to 2005 by ICD-9 codes for preeclampsia (642.4-642.7) and stroke (437.3-437.7) [42]. After confirming with medical record review that the timing of stroke was greater than 6 weeks postpartum at onset, 23 women met the inclusion criteria for having had preeclampsia and a later admission for stroke. The median age at stroke onset was 38 (range 21-62), $18 \%$ or $78 \%$ were African American, and just over half had ischemic stroke (13 ischemic strokes/TIAs, 8 hemorrhagic strokes, and 2 subarachnoid hemorrhages). The majority (70\%) of women were between the ages of 18 and 34 at the time of the last preeclamptic pregnancy $(9 \%<$ age 17 and $22 \%$ over age 35 ). When preeclampsia was divided into mild and severe, there was no association with stroke type, age at stroke onset, or race/ethnicity. In terms of risk factors, $70 \%$ of these women had hypertension, $26 \%$ diabetes, $30 \%$ hyperlipidemia, 59\% smoked tobacco, 13\% used illicit drugs, and $39 \%$ had had miscarriages [42]. In addition, $100 \%$ of the women with hemorrhagic strokes had prepregnancy hypertension, compared with $60 \%$ of the women with ischemic stroke. We also found that the age of onset of hypertension was not associated with the age at stroke [42]. This study was limited because of the small cohort size, the lack of controls, and the inability to track hospitalizations outside of the center where delivery occurred. However, this was a novel longitudinal case series because these data can provide guidance for how additional cohort studies could be ideally designed to investigate the relationships between stroke types and hypertension in women with a history of preeclampsia. For example, based on this database, future cohorts should enroll women who are no more than 5 years from delivery with preeclampsia at baseline. The ongoing HyRAS study is designed to follow women in The Netherlands who are 2.5 years post-preeclampsia for 10 years to track the onset of cardiovascular disease [57]. Future studies that include underrepresented minorities, such as African American women, are needed to better understand the risk of stroke after preeclampsia since these women are at high risk for both preeclampsia and stroke.

\section{Prevention of Stroke in Women}

Aspirin is an important prevention strategy for ischemic stroke in women. Interestingly, it has also been used for prevention for preeclampsia; therefore prevention with aspirin represents another overlap between these two disorders. A recent meta-analysis combined randomized clinical trials of over 11,000 women randomized to low dose aspirin versus placebo to prevent preeclampsia and intrauterine growth restriction [58]. There was a significant reduction in the rate of preeclampsia with low dose aspirin started at 16 weeks

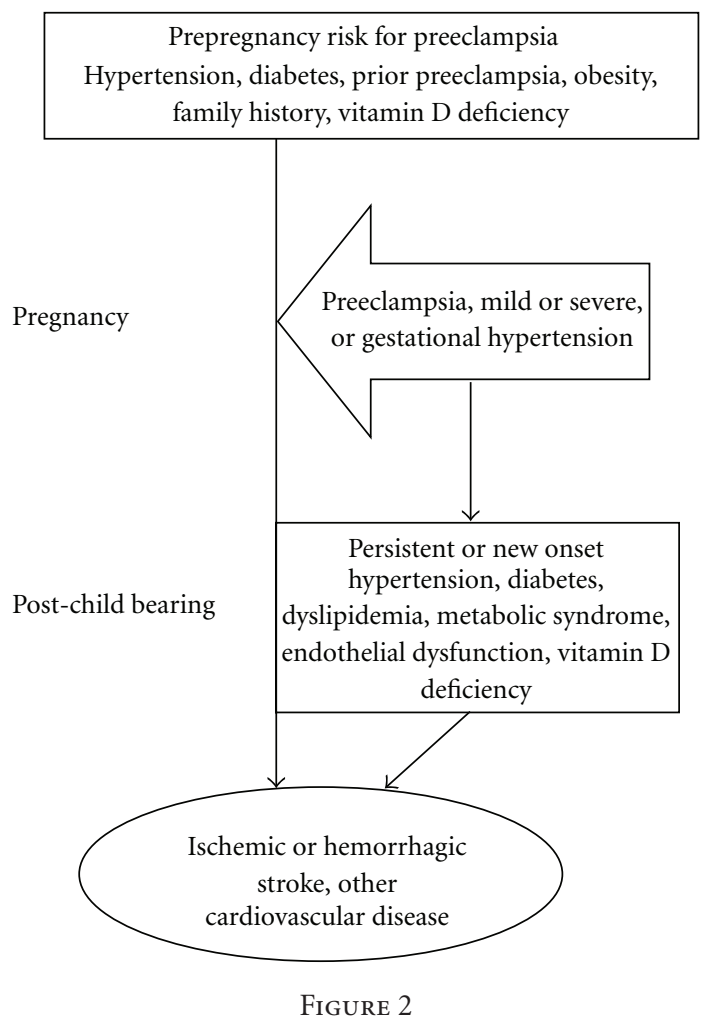

gestation or earlier (RR $0.47,95 \%$ CI $0.34-0.65$ ) in women identified as moderate or high risk. The rate of intrauterine growth restriction was reduced to a similar degree (RR 0.44, 95\% CI $0.30-0.65)$. Since the rate of severe preeclampsia was also reduced, cerebrovascular complications would also theoretically be reduced as well. If aspirin was started after 16 weeks, the reduction in the rate of preeclampsia was no longer significant (RR 0.81, 95\% CI 0.63-1.03) [58]. Caution must be exercised in interpreting this meta-analysis, however, since the inclusion criteria for individual trials of aspirin therapy were heterogeneous. In all studies where aspirin had been started before 16 weeks, only women identified as being at moderate to high risk for preeclampsia were included. Although it appears that some cases of preeclampsia can be prevented in women who are pregnant and at high risk for preeclampsia, the best long-term prevention strategy for these young and middle-aged women is currently unknown. For prevention of stroke later in life, the emphasis should be on recognizing and treating cardiovascular risk factors. It is unlikely that treating all young women with aspirin following the occurrence of preeclampsia without any other risk factors would be dangerous because aspirin increases the risk of hemorrhage. More studies are needed to identify women at early risk of stroke, as well as heart disease, and ultimately assess the risks and benefits of various prevention approaches.

Vitamin D deficiency has recently emerged as an important potentially modifiable risk factor for both preeclampsia and stroke. Women who are deficient in vitamin D before or during pregnancy are at increased risk for preeclampsia [59]. Low serum levels of $25(\mathrm{OH}) \mathrm{D}$ are very common in 
both developed and developing countries. In a populationbased study of vitamin $\mathrm{D}$ intake and risk of preeclampsia, women with an estimated intake of 600-800 IU of vitamin D per day had reduced odds for preeclampsia compared to women with an estimated intake of less than $200 \mathrm{IU} /$ day (OR 0.76, 95\% CI 0.6-0.95). Trials of supplementation with calcium and vitamin $\mathrm{D}$ showed inconsistent effects on the risk of preeclampsia [60]. Large ongoing trials of vitamin D supplementation in pregnancy as a strategy to prevent preeclampsia and other adverse pregnancy outcomes are ongoing. In a recent large electronic medical record database analysis, a $51 \%$ relative increase in stroke prevalence was noted in patients with very low versus normal vitamin $D$ levels $(5.9 \%$ versus $3.9 \% ; P<.0001)$ [61]. The association between vitamin $\mathrm{D}$ deficiency and increased risk for both preeclampsia and stroke again suggests overlapping pathophysiology for these disorders.

\section{Summary}

From a clinical perspective, preeclampsia should be regarded as a risk factor for stroke in pregnancy as well as a harbinger of future cerebrovascular disease, although there are many gaps in our knowledge about who is at risk and when, as well as how to best prevent preeclampsia and stroke in women (Figure 2). There are many opportunities to intervene to reduce morbidity and mortality, but the major obstacle currently is our ability to identify women who are at risk for early cardiovascular disease. Given the overlap in risk factors, the potential for early atherosclerosis, and the evidence of lasting endothelial dysfunction (i.e., subclinical vascular disease) from the literature, several screening tests could be done to risk-stratify women with preeclampsia. It is noteworthy that the spectrum of hypertensive disorders in pregnancy parallels the risk for stroke and cardiovascular disease later in life. Therefore, women should be actively screened for a history of pregnancy complications as part of routine preventive care or during evaluation for stroke risk, even though they are well past their child-bearing years. Using this simple historical information is the first step to risk stratification. Further research on the utility of subclinical vascular measurements, such as cerebral or peripheral vasomotor reactivity, carotid intimal medial thickness, coronary calcification, or clinical and biochemical biomarkers, is needed to identify which women with a history of preeclampsia are at increased risk for future stroke.

\section{References}

[1] C. Jaigobin and F. L. Silver, "Stroke and pregnancy," Stroke, vol. 31, no. 12, pp. 2948-2951, 2000.

[2] A. H. James, C. D. Bushnell, M. G. Jamison, and E. R. Myers, "Incidence and risk factors for stroke in pregnancy and the puerperium," Obstetrics and Gynecology, vol. 106, no. 3, pp. 509-516, 2005.

[3] D. J. Lanska and R. J. Kryscio, "Stroke and intracranial venous thrombosis during pregnancy and puerperium," Neurology, vol. 51, no. 6, pp. 1622-1628, 1998.
[4] L. Bellamy, J. P. Casas, A. D. Hingorani, and D. J. Williams, "Pre-eclampsia and risk of cardiovascular disease and cancer in later life: systematic review and meta-analysis," British Medical Journal, vol. 335, no. 7627, pp. 974-977, 2007.

[5] J. M. Roberts, G. Pearson, J. Cutler, and M. Lindheimer, "Summary of the NHLBI Working Group on research on hypertension during pregnancy," Hypertension, vol. 41, pp. 437-445, 2003.

[6] B. Sibai, G. Dekker, and M. Kupferminc, "Pre-eclampsia," The Lancet, vol. 365, no. 9461, pp. 785-799, 2005.

[7] E. A. Steegers, P. von Dadelszen, J. J. Duvekot, and R. Pijnenborg, "Pre-eclampsia," The Lancet, vol. 376, pp. 631644, 2010.

[8] L. Magee, M. Helewa, J.-M. Moutquin, and P. von Dadlszen, "SOGC Guidelines: diagnosis, evaluation, and management of the hypertensive disorders of pregnancy," Journal of Obstetrics and Gynaecology Canada, vol. 20, supplement, pp. 1-48, 2008.

[9] M. D. Lindheimer, S. J. Taler, and F. G. Cunningham, "Hypertension in pregnancy," Journal of the American Society of Hypertension, vol. 4, no. 2, pp. 68-78, 2010.

[10] E. J. Roccella, "Report of the National High Blood Pressure Education Program Working Group on high blood pressure in pregnancy," American Journal of Obstetrics and Gynecology, vol. 183, no. 1, pp. S1-S22, 2000.

[11] B. M. Sibai, "Chronic hypertension in pregnancy," Obstetrics and Gynecology, vol. 100, no. 2, pp. 369-377, 2002.

[12] K. Duckitt and D. Harrington, "Risk factors for pre-eclampsia at antenatal booking: systematic review of controlled studies," British Medical Journal, vol. 330, no. 7491, pp. 565-567, 2005.

[13] J. I. Einarsson, H. Sangi-Haghpeykar, and M. O. Gardner, "Sperm exposure and development of preeclampsia," American Journal of Obstetrics and Gynecology, vol. 188, no. 5, pp. 1241-1243, 2003.

[14] R. T. Lie, S. Rasmussen, H. Brunborg, H. K. Gjessing, E. LieNielsen, and L. M. Irgens, "Fetal and maternal contributions to risk of pre-eclampsia: population based study," British Medical Journal, vol. 316, no. 7141, pp. 1343-1347, 1998.

[15] J. M. Roberts and C. A. Hubel, "The two-stage theory of preeclampsia: variations on the theme," Placenta, vol. 30, supplement A, pp. S32-S37, 2009.

[16] R. Skjærven, L. J. Vatten, A. J. Wilcox, T. Rønning, L. M. Irgens, and R. T. Lie, "Recurrence of pre-eclampsia across generations: exploring fetal and maternal genetic components in a population based cohort," British Medical Journal, vol. 331, no. 7521, pp. 877-879, 2005.

[17] K. L. Adeney and M. A. Williams, "Migraine headaches and preeclampsia: an epidemiologic review," Headache, vol. 46, no. 5, pp. 794-803, 2006.

[18] C. D. Bushnell, M. Jamison, and A. H. James, "Migraines during pregnancy linked to stroke and vascular diseases: US population based case-control study," British Medical Journal, vol. 338, p. b664, 2009.

[19] S. E. Sanchez, C. Qiu, M. A. Williams, N. Lam, and T. K. Sorensen, "Headaches and migraines are associated with an increased risk of preeclampsia in Peruvian women," American Journal of Hypertension, vol. 21, no. 3, pp. 360-364, 2008.

[20] G. E. Tietjen, M. M. Al-Qasmi, K. Athanas, R. M. Dafer, and S. A. Khuder, "Increased von Willebrand factor in migraine," Neurology, vol. 57, no. 2, pp. 334-336, 2001.

[21] T. Sharshar, C. Lamy, and J. L. Mas, "Incidence and causes of strokes associated with pregnancy and puerperium: a study in public hospitals of Ile de France," Stroke, vol. 26, no. 6, pp. 930-936, 1995. 
[22] J. S. Jeng, S. C. Tang, and P. K. Yip, "Stroke in women of reproductive age: comparison between stroke related and unrelated to pregnancy," Journal of the Neurological Sciences, vol. 221, no. 1-2, pp. 25-29, 2004.

[23] B. T. Bateman, H. C. Schumacher, C. D. Bushnell et al., "Intracerebral hemorrhage in pregnancy: frequency, risk factors, and outcome," Neurology, vol. 67, no. 3, pp. 424-429, 2006.

[24] J. N. Martin, B. D. Thigpen, R. C. Moore, C. H. Rose, J. Cushman, and W. May, "Stroke and severe preeclampsia and eclampsia: a paradigm shift focusing on systolic blood pressure," Obstetrics and Gynecology, vol. 105, no. 2, pp. 246254, 2005.

[25] J. Hinchey, C. Chaves, B. Appignani et al., "A reversible posterior leukoencephalopathy syndrome," New England Journal of Medicine, vol. 334, no. 8, pp. 494-500, 1996.

[26] V. H. Lee, E. F. M. Wijdicks, E. M. Manno, and A. A. Rabinstein, "Clinical spectrum of reversible posterior leukoencephalopathy syndrome," Archives of Neurology, vol. 65, no. 2, pp. 205-210, 2008.

[27] R. B. Schwartz, S. K. Feske, J. F. Polak et al., "Preeclampsiaeclampsia: clinical and neuroradiographic correlates and insights into the pathogenesis of hypertensive encephalopathy," Radiology, vol. 217, no. 2, pp. 371-376, 2000.

[28] G. G. Zeeman, "Neurologic complications of pre-eclampsia," Seminars in Perinatology, vol. 33, no. 3, pp. 166-172, 2009.

[29] L. H. Calabrese, D. W. Dodick, T. J. Schwedt, and A. B. Singhal, "Narrative review: reversible cerebral vasoconstriction syndromes," Annals of Internal Medicine, vol. 146, no. 1, pp. 3444, 2007.

[30] S. Koch, A. Rabinstein, S. Falcone, and A. Forteza, "Diffusionweighted imaging shows cytotoxic and vasogenic edema in eclampsia," American Journal of Neuroradiology, vol. 22, no. 6, pp. 1068-1070, 2001.

[31] R. A. Hajj-Ali, A. Furlan, A. Abou-Chebel, and L. H. Calabrese, "Benign angiopathy of the central nervous system: cohort of 16 patients with clinical course and long-term followup," Arthritis Care and Research, vol. 47, no. 6, pp. 662-669, 2002.

[32] A. B. Singhal, "Postpartum angiopathy with reversible posterior leukoencephalopathy," Archives of Neurology, vol. 61, no. 3, pp. 411-416, 2004.

[33] S. Riskin-Mashiah, M. A. Belfort, G. R. Saade, and J. A. Herd, "Cerebrovascular reactivity in normal pregnancy and preeclampsia," Obstetrics and Gynecology, vol. 98, no. 5, pp. 827-832, 2001.

[34] M. A. Belfort, M. W. Varner, D. S. Dizon-Townson, C. Grunewald, and H. Nisell, "Cerebral perfusion pressure, and not cerebral blood flow, may be the critical determinant of intracranial injury in preeclampsia: a new hypothesis," American Journal of Obstetrics and Gynecology, vol. 187, no. 3, pp. 626-634, 2002.

[35] J. L. James, G. S. Whitley, and J. E. Cartwright, "Pre-eclampsia: fitting together the placental, immune and cardiovascular pieces," Journal of Pathology, vol. 221, no. 4, pp. 363-378, 2010.

[36] A. Hawfield and B. I. Freedman, "Pre-eclampsia: the pivotal role of the placenta in its pathophysiology and markers for early detection," Therapeutic Advances in Cardiovascular Disease, vol. 3, no. 1, pp. 65-73, 2009.

[37] V. Shenoy, K. Kanasaki, and R. Kalluri, "Pre-eclampsia: connecting angiogenic and metabolic pathways," Trends in Endocrinology and Metabolism, vol. 21, pp. 529-536, 2010.

[38] S. Venkatesha, M. Toporsian, C. Lam et al., "Soluble endoglin contributes to the pathogenesis of preeclampsia," Nature Medicine, vol. 12, no. 6, pp. 642-649, 2006.
[39] R. J. Levine, C. Lam, C. Qian et al., "Soluble endoglin and other circulating antiangiogenic factors in preeclampsia," New England Journal of Medicine, vol. 355, no. 10, pp. 992-1005, 2006.

[40] G. C. S. Smith, J. P. Pell, and D. Walsh, "Pregnancy complications and maternal risk of ischaemic heart disease: a retrospective cohort study of 129290 births," The Lancet, vol. 357, no. 9273, pp. 2002-2006, 2001.

[41] D. W. Brown, N. Dueker, D. J. Jamieson et al., "Preeclampsia and the risk of ischemic stroke among young women: results from the stroke prevention in young women study," Stroke, vol. 37, no. 4, pp. 1055-1059, 2006.

[42] M. V. Chireau, C. Bushnell, H. Brown, L. B. Goldstein, and L. A. Bastian, "Pregnancy complications are associated with stroke risk later in life," Annals of Neurology, vol. 58, supplement 9, p. S41, 2005.

[43] B. J. Wilson, M. S. Watson, G. J. Prescott et al., "Hypertensive diseases of pregnancy and risk of hypertension and stroke in later life: results from cohort study," British Medical Journal, vol. 326, no. 7394, pp. 845-849, 2003.

[44] H. U. Irgens, L. Reisæter, L. M. Irgens, and R. T. Lie, "Long term mortality of mothers and fathers after pre-eclampsia: population based cohort study," British Medical Journal, vol. 323, no. 7323, pp. 1213-1217, 2001.

[45] R. J. Kaaja and I. A. Greer, "Manifestations of chronic disease during pregnancy," Journal of the American Medical Association, vol. 294, no. 21, pp. 2751-2757, 2005.

[46] L. S. Jonsdottir, R. Arngrimsson, R. T. Geirsson, H. Sigvaldason, and N. Sigfusson, "Death rates from ischemic heart disease in women with a history of hypertension in pregnancy," Acta Obstetricia et Gynecologica Scandinavica, vol. 74, no. 10, pp. 772-776, 1995.

[47] J. G. Ray, M. J. Vermeulen, M. J. Schull, and D. A. Redelmeier, "Cardiovascular health after maternal placental syndromes (CHAMPS): population-based retrospective cohort study," The Lancet, vol. 366, no. 9499, pp. 1797-1803, 2005.

[48] P. Hannaford, S. Ferry, and S. Hirsch, "Cardiovascular sequelae of toxaemia of pregnancy," Heart, vol. 77, no. 2, pp. 154158, 1997.

[49] E. Kajantie, J. G. Eriksson, C. Osmond, K. Thornburg, and D. J. P. Barker, "Pre-eclampsia is associated with increased risk of stroke in the adult offspring the helsinki birth cohort study," Stroke, vol. 40, no. 4, pp. 1176-1180, 2009.

[50] N. Sattar, J. Ramsay, L. Crawford, H. Cheyne, and I. A. Greer, "Classic and novel risk factor parameters in women with a history of preeclampsia," Hypertension, vol. 42, no. 1, pp. 3942, 2003.

[51] H. Laivuori, M. J. Tikkanen, and O. Ylikorkala, "Hyperinsulinemia 17 years after preeclamptic first pregnancy," Journal of Clinical Endocrinology and Metabolism, vol. 81, no. 8, pp. 2908-2911, 1996.

[52] M. Vickers, I. Ford, R. Morrison et al., "Markers of endothelial activation and atherothrombosis in women with history of preeclampsia or gestational hypertension," Thrombosis and Haemostasis, vol. 90, no. 6, pp. 1192-1197, 2003.

[53] J. C. Chambers, L. Fusi, I. S. Malik, D. O. Haskard, M. De Swiet, and J. S. Kooner, "Association of maternal endothelial dysfunction with preeclampsia," Journal of the American Medical Association, vol. 285, no. 12, pp. 1607-1612, 2001.

[54] P. K. Agatisa, R. B. Ness, J. M. Roberts, J. P. Costantino, L. H. Kuller, and M. K. McLaughlin, "Impairment of endothelial function in women with a history of preeclampsia: an indicator of cardiovascular risk," American Journal of Physiology, vol. 286, no. 4, pp. H1389-H1393, 2004. 
[55] J. Blaauw, R. Graaff, M. G. Van Pampus et al., "Abnormal endothelium-dependent microvascular reactivity in recently preeclamptic women," Obstetrics and Gynecology, vol. 105, no. 3, pp. 626-632, 2005.

[56] E. G. H. J. Martens, L. L. H. Peeters, E. D. Gommer et al., "The visually-evoked cerebral blood flow response in women with a recent history of preeclampsia and/or eclampsia," Ultrasound in Medicine and Biology, vol. 35, no. 1, pp. 1-7, 2009.

[57] W. Hermes, A. Franx, M. G. van Pampus et al., "10-Year cardiovascular event risks for women who experienced hypertensive disorders in late pregnancy: the HyRAS study," BMC Pregnancy and Childbirth, vol. 10, article 28, 2010.

[58] E. Bujold, S. Roberge, Y. Lacasse et al., "Prevention of preeclampsia and intrauterine growth restriction with aspirin started in early pregnancy: a meta-analysis," Obstetrics and Gynecology, vol. 116, no. 2 PART 1, pp. 402-414, 2010.

[59] L. M. Bodnar, J. M. Catov, H. N. Simhan, M. F. Holick, R. W. Powers, and J. M. Roberts, "Maternal vitamin D deficiency increases the risk of preeclampsia," Journal of Clinical Endocrinology and Metabolism, vol. 92, no. 9, pp. 3517-3522, 2007.

[60] H. Barrett and A. McElduff, "Vitamin D and pregnancy: an old problem revisited," Best Practice \& Research Clinical Endocrinology \& Metabolism, vol. 24, pp. 527-539, 2010.

[61] J. L. Anderson, H. T. May, B. D. Horne et al., "Relation of vitamin $\mathrm{D}$ deficiency to cardiovascular risk factors, disease status, and incident events in a general healthcare population," American Journal of Cardiology, vol. 106, no. 7, pp. 963-968, 2010. 


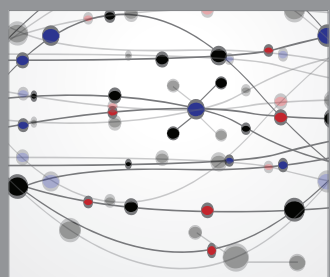

The Scientific World Journal
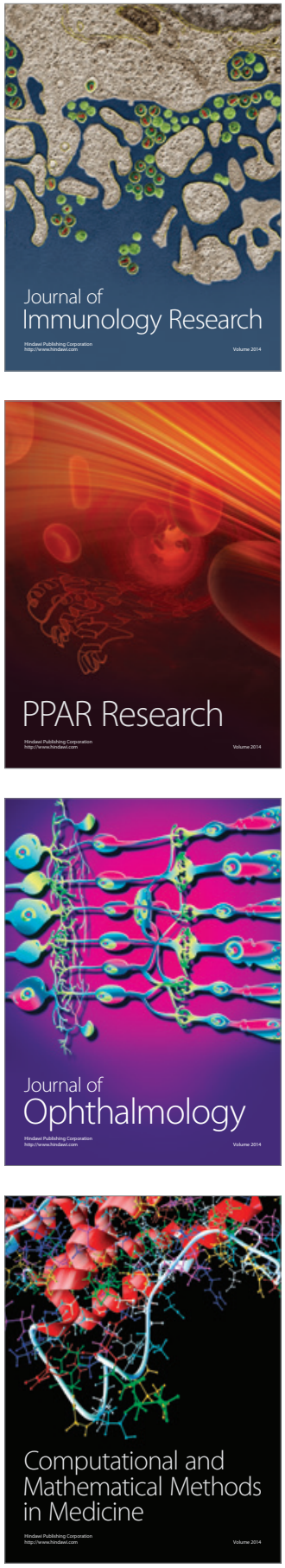

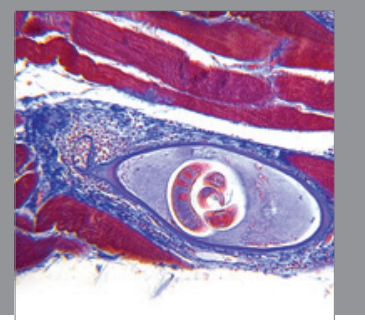

Gastroenterology

Research and Practice
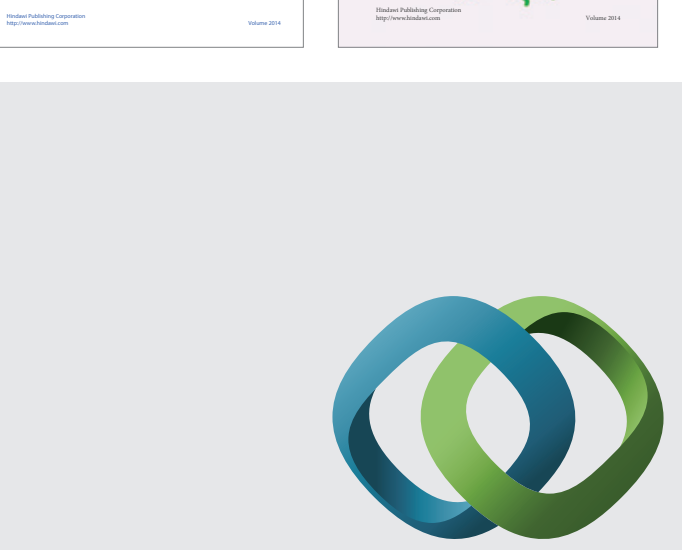

\section{Hindawi}

Submit your manuscripts at

http://www.hindawi.com
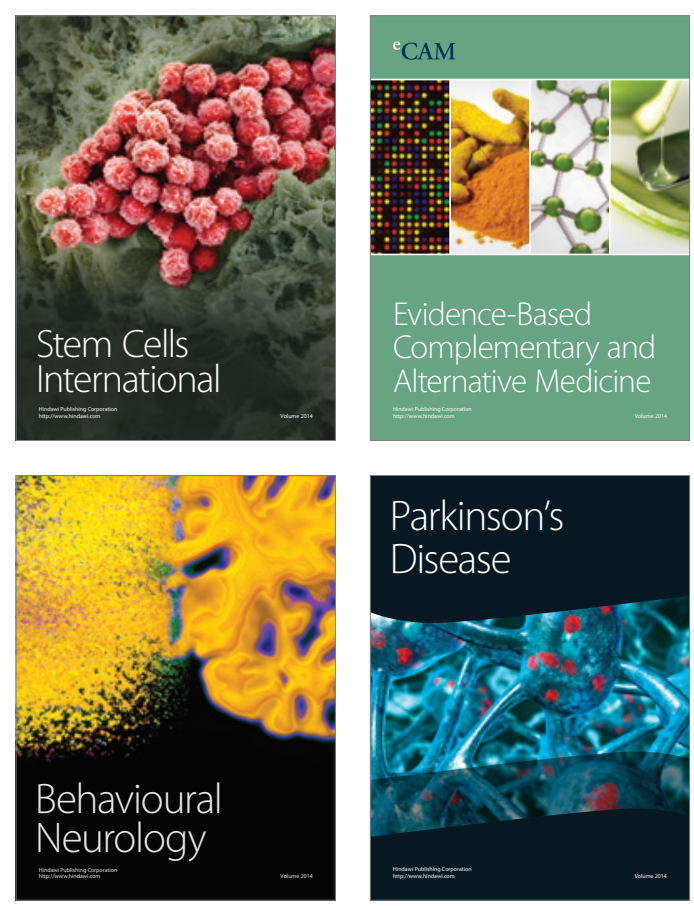

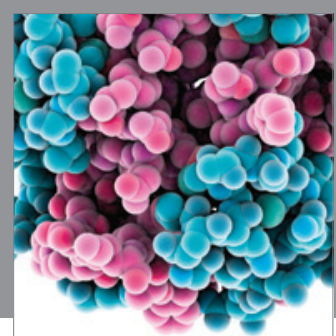

Journal of
Diabetes Research

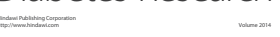

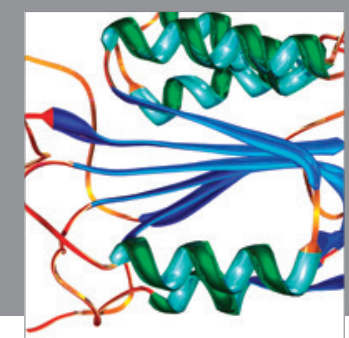

Disease Markers
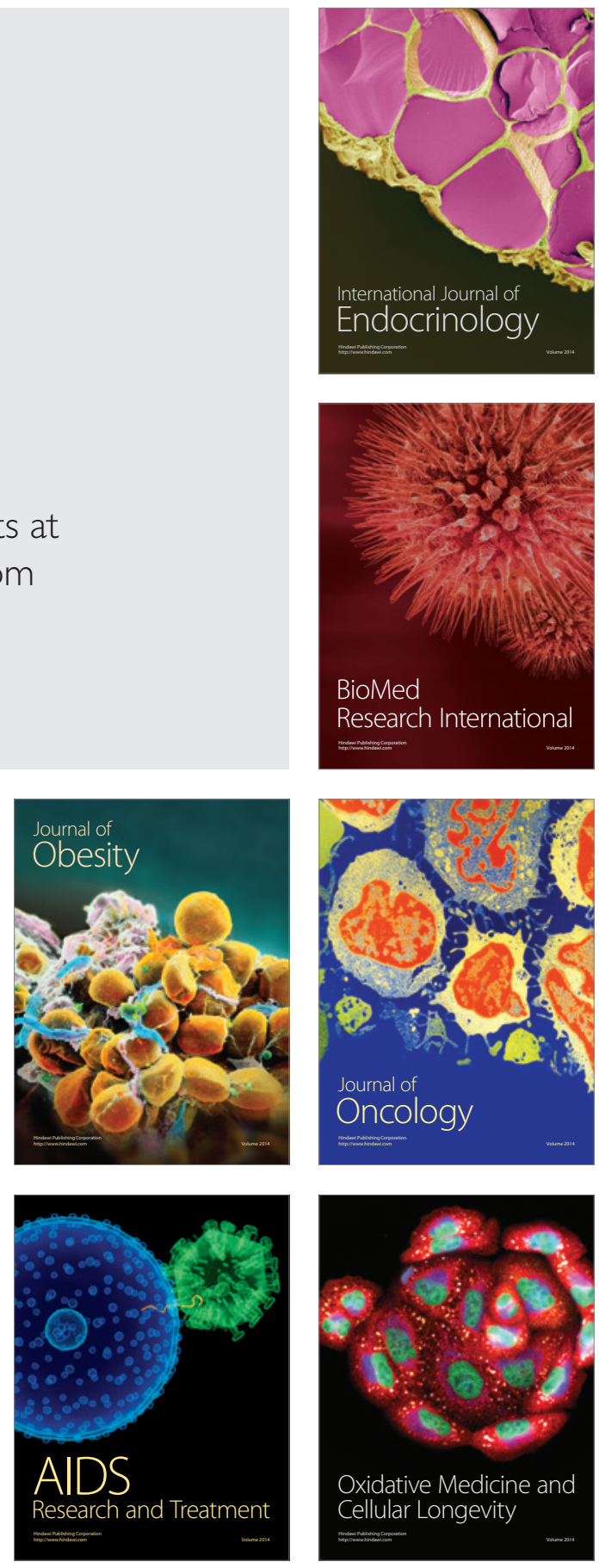